\title{
Rethinking the Specious Present
}

\author{
Simon Prosser
}

\section{Introduction}

In this chapter I shall argue that despite its current popularity the doctrine of the specious present, or at least every current version of it, should be rejected. ${ }^{1}$ In its place I propose two different accounts, which deal with experiences of two different kinds of change. The first is what I shall call the dynamic snapshot theory, which accounts for the way we experience continuous changes such as motion and other motion-like phenomena. The second account deals with the way we experience discontinuous changes, those for which there is no finite rate of change. In defending both accounts, but especially the latter, I shall argue that much of the current debate implicitly presupposes a problematic Cartesian view about the nature of conscious experience. If this view is rejected - as I think it should be - then a different kind of account emerges that avoids commitment both to the specious present and to its main current rival, the cinematic view.

\section{The Current Orthodoxy}

A significant majority of philosophers writing about temporal experience today accept one or another version of the doctrine of the specious present, according to which

\footnotetext{
${ }^{1}$ A more detailed account of the ideas presented in this chapter can be found in Prosser 2016: chapter 5 .
} 
conscious experiences have temporally extended contents. That is to say, they hold that the experienced present consists not of an instant, but of an extended interval of time. The duration of this interval is usually taken to be small: around half a second, say. There are also those who, following James (1890), use the phrase "specious present" to denote a temporal interval of several seconds. In such cases, however, it usually turns out that what they have in mind is a kind of "psychological present" associated with short-term memory. I have nothing to say about the latter notion of the specious present; I shall be concerned only with the former. The question before us is whether, or in what sense, conscious perceptual experiences - experiences with a phenomenology, or "something that it is like" (in Nagel's (1974) sense) - have a short but temporally extended content.

There are two main versions of the doctrine which, following Dainton (2008, 2010), I shall call the retentional and extensional models. Suppose the duration of the specious present is $n$ seconds. According to the retentional model (Broad 1938; Lee 2014), there is no reason to expect that the experience itself should occur over a period of $n$ seconds; the experience need not be, and typically is not, extended to the same degree as its content. Traditionally, the experience itself was assumed to take place instantaneously; however Geoffrey Lee (2014) has made a strong case for the view that the experience itself may take some time to occur. Crucially, however, even on Lee's "extended atomism" version of retentionalism, it is not the case that an experience of the short sequence $\mathrm{A}-\mathrm{B}-\mathrm{C}$ involves the subject experiencing $\mathrm{A}$, then experiencing $B$, then experiencing $C$. Instead, there is a single experience of the sequence A-B-C. According to the extensional model (Foster 1979; Foster 1982: chapter 16; Foster 1991: 246-50; Dainton 2000; 2001; 2008; Hoerl 2009; 2013; Phillips 2010; 2011; 2014; Rashbrook 2013), by contrast, the temporal structure of the experience matches the temporal structure of its content. Thus if the content of the experience is an interval of time $n$ seconds long, then the experience itself lasts $n$ seconds. Moreover, most extensionalists also assume that the internal structure of the experience matches the structure of the experienced events; if the content of the specious present is the sequence $\mathrm{A}-\mathrm{B}-\mathrm{C}$ then the subject experiences $\mathrm{A}$, then experiences $\mathrm{B}$, then experiences $\mathrm{C}$. 
The most discussed competitor to the doctrine of the specious present is the cinematic model (defended by Chuard 2011. The name is used in Dainton 2010 and elsewhere.) According to the cinematic model, experience consists of a series of "static snapshot" experiences, the content of which is a single instant of time. Change, on this view, is experienced only indirectly, through the combination of a current experience with a very recent memory.

\section{Arguments for the Specious Present}

The literature on temporal experience contains surprisingly few direct arguments in favor of the specious present. The most common type of argument appeals to the fact that we can perceive change. As C. D. Broad observed, there is at least a strong prima facie case for thinking that we do perceive certain kinds of change, such as motion, rather than inferring them from a combination of immediate experience and episodic memory:

...We do not merely notice that something has moved or otherwise changed; we also often see something moving or changing. This happens if we look at the second-hand of a watch or look at a flickering flame. These are experiences of a quite unique kind; we could no more describe what we sense in them to a man who had never had such experiences than we could describe a red colour to a man born blind. It is also clear that to see a second-hand moving is a quite different thing from "seeing" that an hour-hand has moved. In the one case we are concerned with something that happens within a single sensible field; in the other we are concerned with a comparison between the contents of two different sensible fields. (Broad 1923: 351)

It does indeed seem that motion perception has a robust phenomenology; it involves a phenomenological element that is quite unlike anything that can be encountered in the perception of a static scene. Many philosophers have dismissed the Cinematic model, with its "static snapshot" content, as incapable of accounting for this 
phenomenology, and have assumed that it can only be accounted for by some version of the doctrine of the specious present. The idea seems to be that because a single specious present would contain the moving object at different positions at different times, motion would be part of the content of the experience.

Sometimes a more direct argument is given. This usually takes something like the following form. Motion, and indeed change of any other kind, essentially takes time. An experience whose content consisted in the state of the world at an instant could only have in its content that which could occur within the instant. It thus could not include anything essentially extended in time. Therefore, given that motion essentially takes time but can be perceived, the content of an experience cannot be an instant. ${ }^{2}$

Similar arguments can be given by appealing to anything perceptible but essentially extended in time. Consider sounds, for example. There are differing views about what one is aware of when one hears a sound. Suppose, for present purposes, that when one hears a sound, the object of one's experience is an objective entity such as a process or event - the fingernails scraping across the blackboard, the wind moving through the trees, the vibration of the violin string, and so on. ${ }^{3}$ It seems plausible that a process essentially takes time; there is no such thing as an instantaneous process. In that case, it might be argued, since the object of auditory experience is essentially temporally extended, auditory experience cannot have an instantaneous content.

Arguments of this kind are not sound; they fail because they mistakenly assume that an experience with an instantaneous content can have, in its content, only that which could occur instantaneously. This is simply false, for extended processes nevertheless have instantaneous parts, even if the nature of such parts depends on what occurs at other times. All that can be concluded from such arguments is that the surrounding temporal context must be detected by the system that produces the experience. Rather than continue to address this point in the abstract, however, I shall illustrate it by sketching a theory, which I shall call the dynamic snapshot theory, that allows for motion to be perceived without recourse to the specious present.

\footnotetext{
${ }^{2}$ A clear example of this kind of argument is given by Grush (2007: 1), but similar arguments are encountered frequently.

${ }^{3}$ See Casati and Dokic 1994, Pasnau 2007 and O'Callaghan 2007 for theories of sound broadly of this kind.
} 
One small concession must be made. Perception has a finite resolution, and this affects the degree to which experience can be said to have precisely one time as its content. By analogy, consider spatial perception. All else being equal, below a certain size, it is impossible to tell which of two very small objects is the smallest; all extremely small objects will look the same, at least insofar as they reflect or produce the same amount of light. This means that using vision alone it would be impossible to detect the difference between a point-sized object and a small but slightly extended one. Something similar is true of temporal experience: below a certain temporal extension, one cannot detect differences. ${ }^{4}$ It does not follow from this, however, that experience has a temporally extended content in any interesting sense. It follows only that it is indeterminate, to a small degree, which moment in time is the content of the experience. If the doctrine of the specious present is to account for the experience of change then it requires instead a duration long enough to include different, temporally discriminable states.

\section{The Dynamic Snapshot Theory}

Suppose that having a perceptual experience were like being aware of a kind of internal picture. If that picture were a snapshot of a single instant, it would contain no motion. Objects would be depicted as occupying a single locations in space; there would be no way to tell which objects were moving, or in what way. A photograph taken with an exposure time greater than zero would show objects as slightly blurred due to their motion, but if the picture really depicted a single instant then there would be no clues of this sort. In order to judge that there was motion, one would have to compare the picture with another picture taken at a different time, showing the objects in different places.

But conscious experience is not at all like an awareness of an inner picture. To think of experience in that way would be to fall into a problematic Cartesian view of

\footnotetext{
${ }^{4}$ For vision the threshold for detecting distinct stimuli is $20 \mathrm{~ms}$, for audition it is $2-3 \mathrm{~ms}$, and for touch it is $10 \mathrm{~ms}$ (see Ruhnau 1995). See also Pockett 2003. In all sensory modalities there is the same threshold for the discrimination of temporal order: around 20-40 ms (Hirsh and Sherrick 1961, Pöppel 1997).
} 
the conscious mind. I shall say more about this in the final section, but for now note the obvious difficulty with the "inner picture" model: if perception of the external world involved awareness of an inner picture, an account would have to be given of this "awareness". But this could not be accounted for in the same way as perceptual awareness of the external world, for an infinite regress of inner pictures would threaten. If it worked in some other way, however, then it is unclear why that account would not already explain awareness of the external world, removing the need for the inner picture.

We can make better sense of experience by thinking in terms of the content of the experience. The content of an experience is what the experience is of. When you see a tree next to a hill, the content of your experience is that there is a tree next to a hill (along with your own perspective on the scene and whatever further details are perceived). Sometimes philosophers claim that experiences have representational contents. The main point of this is to allow that the experience could be falsidical; it could have the content that there is a tree next to a hill even if this were not the case. But for present purposes we can side-step the debate over whether experiences are representational by focusing on the veridical case, and using the word "content" as neutral with regard to the notion of representation (different theories of perception, such as naïve realism and intentionalism, will still say different things about falsidical cases).

Now, the "inner picture" model makes it tempting to think that if the content of an experience concerned only a single time, it could not include motion. But, as Broad notes in the passage quoted above, "we also often see something moving or changing". "Moving" is a state that something can be in at an instant, even though it can only be in that state by virtue of being in other places at other times. So the motion of an object, including its direction and rate, could be part of the content of an experience, even if the content of that experience concerned only what was the case at one specific time. Suppose that a perceived object, $O$, were moving with velocity $v$, where " $v$ " is a vector (that is, it encodes both the rate and direction of motion). Then the content of the experience could be: $O$ is moving with velocity $v$. A corresponding claim could be made for any other case in which there was an on-going process whose state could be specified by a vector at a single time, even if the existence of the state at that time was 
metaphysically dependent on what was the case at other times. Cognitive scientists sometimes refer to all such continuous perceptible changes, such as the steady dimming of a light, the shifting of a color hue or the rise and fall in the pitch or volume of a sound, as "motion" (see Rensink 2002). I shall refer to them as continuous changes, or sometimes as motion-like changes. ${ }^{5}$

It would, of course, take time for the brain to detect motion, for example by comparing patterns of retinal stimulation at different times. But it clearly does not follow from this that the resulting experience must have a temporally extended content. The necessary properties of the stimulus can differ from those of the resulting experience.

I shall call the theory outlined above the dynamic snapshot theory. ${ }^{6}$ It is a "snapshot" theory because it accounts for the experience of motion without appeal to a specious present, but it is very different from the cinematic or "static snapshot" theory. I agree with those who hold that the latter theory cannot adequately account for the phenomenology of motion experience, or the experience of other motion-like changes.

The dynamic snapshot theory gains some plausibility from various empirical sources. Perhaps one part of the intuition that snapshot experiences can only be static is connected with the idea that for there to be an experience of change, the content of one's experience must itself change over time. But this appears to be false; there are many examples of motion illusions in which motion is experienced despite the fact that no part of the content of the experience changes (apart from the time itself). The best-known example is the waterfall illusion (Wohlgemuth 1911) in which, after a period of looking at steady motion such as motion of the water in a waterfall, when

\footnotetext{
${ }^{5}$ What about sounds? If the perceived object of auditory experience is an event or a process, as suggested above, could this be represented by a vector? I see no reason why not, though I have space only for a very limited explanation here. At any given moment the auditory signal can be represented by a frequency spectrum, representing the intensity at different wavelengths of sound at a given time. This corresponds to the frequency distribution of a perceived physical vibration (the sound source) at that time, and this could be represented by a vector.

${ }^{6}$ Something similar is briefly outlined by Robin Le Poidevin (2007: 88-92), who also makes a similar appeal to the waterfall illusion (see below). While writing this chapter, subsequent to completing Prosser 2016 (in which I also used the name 'dynamic snapshot theory'), I discovered that Valtteri Arstila (2016) has also used the same name for a similar view (Arstila's ambitions for the dynamic snapshot theory appear to be a little more extensive than mine; but he does not distinguish sharply between continuous and discontinuous change, so I'm not certain of his view regarding the latter. Arstila also has an interesting take on the much-discussed 'postdiction' phenomena.) I have also heard Bradford Skow suggest something similar to the dynamic snapshot theory in a talk in June 2015.
} 
the subject looks at a stationary scene, the scene appears to move in the opposite direction to the motion that had been perceived. Yet subjects report that the positions of objects in the scene do not appear to change. There are many similar phenomena: see for example the "fine grain motion illusion" (Exner 1875, Thorson, Lange and Biederman-Thorson 1969), in which motion is experienced between points that are indistinguishably close together, or the phenomenon in which motion is observed in a two-frame sequence where one frame is below the threshold for pattern detection (Morgan and Cleary 1992). In all such cases motion is experienced even though relevant aspects of the content of the experience remain constant. If experience were anything like an encounter with an inner picture, such cases ought not to be possible.

The standard explanation for the waterfall illusion appeals to neural adaptation that leads to a shift in the base activation level in populations of neurons that are associated with motion detection in early visual processing (see Anstis, Verstraten and Mather 1998). Due to this shift, the adapted neurons indicate motion in the absence of a moving stimulus. The suggestion is then that the brain processes information in a variety of different streams, each of which computes something different. The content of conscious experience comprises various different contents thus computed. Sometimes, due to neural adaptation, those processes produce the result that an object is moving, despite there being no variation over time in its independently computed location.

Whether or not this is the correct explanation for the waterfall illusion, the very possibility of such an explanation illustrates the possibility that computational processes in the visual system could yield the content that the object is moving (with velocity $v$ ) at a specific time.

According to the dynamic snapshot theory, what is experienced as happening at $t$ is in no part constituted by what is experienced as happening at other times close to $t$. This conflicts with what is said by certain other theories (e.g. the extensional account defended by Phillips (2010; 2011; 2014), if I understand it correctly). But it does not follow that the dynamic snapshot theory entails the possibility a subject who experiences motion at $t$ and has no experience as of any other times close to $t$. For all the dynamic snapshot theory says, there might be other reasons for denying such a possibility (see Prosser 2016: 148-154 for related discussion). 


\section{Discontinuous changes}

Although the dynamic snapshot theory gives a straightforward account of the experience of motion and other continuous changes, it cannot explain the experience of discontinuous changes. These are changes such that there is one state of affairs up to and including time $t$, but a different state of affairs at all times thereafter, and hence no finite rate of change between the states. An example would be a light that illuminated or changed color instantly, or at least quickly enough that the human visual system would not distinguish the change from one that was genuinely instantaneous. Suppose the light were red at all times up to and including $t$, and green at all times thereafter. There would be no time at which the change was taking place; the light would always be determinately one color or the other, and would never be in a state of transition between the two. (Perhaps no real light changes color instantaneously. But there are real lights that change color sufficiently quickly to be indistinguishable, to our limited visual systems, from a genuinely instantaneous change. See Prosser 2016: 128-9 for further discussion of this issue.) Consequently, if the content of experience included only a single instant of time, it would be impossible for the change of color to be part of the content of any experience. Yet there does seem to be some sense in which we can see a light instantly change color.

One option at this point would be to regard discontinuous change as a counterexample to the dynamic snapshot theory, and consequently to reject it. I believe, however, that a good case can be made for giving two different accounts, one for the experience of continuous changes - the dynamic snapshot theory - and a different account for the experience of discontinuous changes. It seems plausible that the human brain should detect different kinds of changes in different ways. Given the finite nature of human beings, those systems that detect the rate of a continuous change must have a threshold beyond which the rate is too quick for the brain to measure. It follows that discontinuous changes cannot be detected in the same way. But it is nonetheless useful to be able to perceive rates of change in those cases in which the rate falls within the measurable range. Many organisms may also have a 
need to detect discontinuous changes. Consequently it should not seem surprising if different kinds of change are detected using different systems. I shall suggest below that the empirical evidence weighs in favor of this. Finally, there is arguably a significant phenomenological difference between experiences of continuous and discontinuous changes. When one perceives a continuous change such as motion there is a robust phenomenology associated with the change, as illustrated by Broad's comments about the second hand of the watch. It is robust enough to be straightforwardly attended to. But the phenomenology of discontinuous change seems far more elusive; by the time there is anything to notice, the change, and the experience of it, has already taken place.

I shall develop a tentative model of discontinuous change perception that will initially appear to resemble the cinematic model. In the next section, however, I shall argue that the cinematic, retentional and extensional models can only be distinguished from one another if a problematic Cartesian assumption is made concerning the nature of conscious experience. If that assumption is rejected, as I think it should be, then we arrive at a better theory according to which the question of whether experience has a temporally extended content should be rejected rather than answered.

Consider how the human visual system could detect discontinuous changes (I shall concentrate on visual examples, but there is no obvious reason why a broadly similar story could not be told for other sensory modalities). One very simple model would say that all of the perceived information in a scene is retained in some kind of shortterm memory and compared with the scene a moment later. This would require a lot of memory capacity, and a lot of information processing. The result would be that any change in the visible properties of a scene should be noticed. But this is not the case. Studies of change blindness (Rensink, O'Regan and Clark 1997, Simons and Levin 1997) show that when a change is sufficiently slow, or is masked by a flicker in all or a large part of the scene, the subject may fail to notice even quite a large change. Consequently most cognitive scientists have rejected the simple model just outlined (see Rensink 2002: 260-4 for further details of empirical evidence against the model).

Instead, the following model has become fairly widely accepted among empirical scientists working on change detection (see Rensink 2002 and O'Regan 2002). Among other things, the early stages of visual processing detect visual transients, rapid 
changes in the luminance or color of the retinal image. At the stage of processing at which transients are detected, no details of the change itself have yet been computed. The function of the detection of the transient appears to be to automatically draw the subject's visual attention to the location of the transient. Ordinarily, where there is no attention, experiential information is not retained. One function of attention, however, is to cause information concerning the attended location to be briefly retained in a short-term memory buffer. This in turn makes it possible to compare the current scene with what went immediately before in the region in which the transient was detected. Consequently, when a transient draws attention to a location, the subject typically notices what has changed at that location. At unattended locations, however, a change may be missed, because information about the preceding state at that location is discarded before the subject can become aware that anything has changed. When changes are slow, no transient is produced; and when there is a flicker, the visual system is swamped with transients, making it relatively unlikely that attention will be directed to the location at which the change occurs. This is thought to be why change blindness occurs.

This empirical model of change detection fits well with the phenomenology of discontinuous change. My hypothesis is that when a transient is detected, this produces an element of experience with a content something like "a change has just occurred here" or perhaps "a change is occurring here", where "here" refers to the perceived location of the transient. This helps explain the familiar experience of noticing a brief change in peripheral vision without being able to say exactly what changed. ${ }^{7}$ It also, I suggest, accounts for the fact that when one does notice a discontinuous change, there is something phenomenological associated with the change, but something rather less robust than the phenomenology of continuous changes. This is associated with a feeling that the perceived object was in a different state a moment before (a state which one may be able to recall).

Given the appeal to a short-term memory that is compared with current experience, the account of the experience of discontinuous change suggested here does have a certain amount in common with the cinematic model. Note that the memory in

\footnotetext{
${ }^{7}$ There is good independent evidence that change detection (i.e. detecting that there was a change) is distinct from change identification (i.e. identifying what changed). See for example Turatto and Bridgeman 2005, especially p. 596.
} 
question is unlikely to be long-term episodic memory; the case is not like Broad's example of seeing that the hour hand has moved by remembering that it was in a different place at an earlier time. In the model under discussion, it is not supposed that there is any kind of conscious inference involved. The short-term memory buffer in which the earlier state of the object is stored presumably allows a more direct kind of comparison. I shall now argue, however, that the distinction between the cinematic, extensional and retentional models should itself be rejected, and the theory just proposed should be reconstrued accordingly.

\section{Rejecting Cartesian Qualia}

Do the cinematic, retentional and extensional models differ empirically? If they did, then it would be an empirical question which, if any, was correct. But it is not clear that they do differ empirically. At any rate, each seems committed to the following claims about the information processing involved when someone perceives a change and reports on it. For simplicity, consider a simple discontinuous change, as described above: an object is red at all times up to and including $t$, and green at all times thereafter. Each theory must acknowledge that firstly information must be received to the effect that the object is red, and that this information must still be present in the brain when it subsequently detects that the object is green. These two pieces of information then interact to produce a verbal report to the effect that the object changed from red to green. According to the cinematic model the information is retained as a short-term memory, which is compared with current experience. According to the retentional model, the information that the object was red at times up to $t$ is retained and combined with the information that the object was green thereafter to produce an experience whose content is that the object changed from red to green. The experience of the change then produces the verbal report. Finally, the extensional theory holds that the object is experienced as red, then experienced as green, and the temporally extended experience that encompasses both states is an 
experience of the object changing from red to green. ${ }^{8}$ Again, this temporally extended experience somehow leads to the verbal report, though in order to do so some trace of the "red" experience must be retained for long enough to influence the report. ${ }^{9}$

So, on the face of it, all three theories tell much the same story about information processing; they all agree that information about the "red" state of the object must be retained and combined with information about the "green" state in order to produce the verbal report that the object changed from red to green. At any rate, I shall take it as a working hypothesis that all of these theories tell the same story about the way information is processed during the perception of discontinuous change. If this turns out to be incorrect then it should be possible, in principle, to rule out at least one theory on empirical grounds. But let us suppose that this is not so; the theories are empirically equivalent. The difference between them, then, seems to consist entirely in what they say about conscious experiences. Given a flow diagram of the information processing that is involved in experiencing discontinuous change, the difference between the theories will amount to differences in which parts of the diagram should be designated as constituting (or causing) conscious experiences, and which should be designated as memories. ${ }^{10}$ To put this another way, the difference will consist in where the qualia appear in the diagram.

This assumes that there are such things are qualia. Daniel Dennett $(1988 ; 1991)$ has argued, however, that qualia, as commonly construed, are a myth (he does not, of course, deny that there are conscious experiences in some broad sense, or that there is "something that it is like" to have a conscious mind). The notion of qualia to which Dennett is opposed says that qualia are ineffable, intrinsic, private and directly or immediately apprehensible. This, for Dennett, is associated with the notion of the mind as a Cartesian theatre, where the qualia are like actors on a stage, appearing before an audience (the "self"), such that the qualia enter and leave the stage at a definite time, and there are definite facts about such matters as what counts as the cessation of the conscious experience and the beginning of the reaction to it.

\footnotetext{
${ }^{8}$ It is not clear to me what makes it the case that the temporal stages of the experience are parts of a unified experience, on the extensional model, or how this would account for the phenomenology. See Prosser 2016: 143-8 for details.

${ }^{9}$ Lee (2014) raises a similar point as an objection to the extensional model; see his discussion of the "trace integration" argument.

${ }^{10}$ See Prosser 2016: 156-7 for diagrams of this kind.
} 
I shall not rehearse Dennett's arguments here. But if his characterization of the problematic assumption is even roughly right (and we need not accept every detail of his description of the putative qualia to think so), then it is not clear that the distinction between the three main models of temporal experience can be sustained. Let us start by considering the difference between the cinematic and retentional models. Suppose that a change from A to B is experienced. The former theory says that change is experienced because a current perception of $\mathrm{A}$ is combined with a very recent short-term memory of $\mathrm{B}$; the latter says that change is experienced because a single experience has the content that $\mathrm{A}$ is followed by $\mathrm{B}$. Provided we restrict attention to discontinuous changes, is there any way that introspection could tell us which of these theories was correct? How would we know what to look for? It does not seem at all clear that we have any capacity to distinguish, introspectively, between the different models. Indeed if both models agree on all matters of information processing, as I suggested above, then both models should predict exactly the same verbal reports of conscious experiences. If we then compare either of these two models with the extensional model, we find exactly the same situation: introspection cannot, and should not, be able to settle which model is correct. ${ }^{11}$

It will nevertheless seem to many people that there has to be an answer. The three models disagree concerning when the various experiences occur, or when a given experience begins and ends. According to the cinematic and extensional models, the subject experiences A, then experiences B. But according to the cinematic model this consists in a sequence of independent snapshot experiences, whereas according to the extensional model it is a single experience that takes time to occur. According to the retentional model, $\mathrm{A}$ and $\mathrm{B}$ both belong to the content of a single experience, with no sense to be made of the claim that the experience of $A$ precedes the experience of $B$.

All of this makes sense only if it is assumed that a conscious experience begins and ends at a precise, determinate moment in time - that incoming information crosses a "finish line" at some determinate point and thereby enters consciousness, and then exits at a similarly determinate moment. This is very much the notion of the

\footnotetext{
${ }^{11}$ Phillips (2014) does suggest that introspection favors extensionalism, but his argument for this interpolates from long-term to short-term experience in a way that strikes me as problematic. See Prosser 2016: 143-6 for discussion. However Phillips (2010) does also briefly express some doubts, not entirely dissimilar to those expressed here, about how much of a difference there really is between the main competing theories.
} 
Cartesian theatre; a given actor (i.e. a quale) is either on the stage or is not, and must enter and leave the stage at determinate moments in time. If one accepts this, then one must hold that one of the three theories of temporal experience is correct. But why must we accept it? Presumably it is part of an implicit theory that has qualia as part of its ontology. If we reject the theory, we may also reject the ontology. I suggest that we have no good reason to accept this theory. It entails that there are facts about matters about which we have no reason to believe that there are facts. The three models do not differ in what they say about information processing, or in what they say about introspection (or so I am assuming). So there is no observation, of any kind, that could determine which was correct.

Most philosophers these days hold that we should not, in general, reject ontological claims for purely verificationist reasons. It is not always the case that if we cannot detect the difference empirically, then it is meaningless to suppose that there is a difference. But conscious experience may be an exception to this. Qualia are supposed to be properties of experiences whose whole essence is to determine "what it is like" for the subject who has the experience. But can there be facts about what it is like that transcend one's ability to introspect, even in principle? The issue is not whether introspection is an infallible guide to conscious experience. Clearly it is not; there are many examples that show that subjects can make mistaken judgments about their conscious experiences. But to say that introspective error is possible is one thing; it is quite another thing to claim that there could be intelligible differences in the way we model conscious experiences, such as those described above, such that it would be impossible in principle for introspection ever to discern which model was correct. When it comes to conscious experience, if we cannot tell the difference, even in principle, then we should be deeply suspicious of the claim that there really is a difference.

Perhaps there could be some kind of a priori argument that would show one theory to account for experience better than another. Perhaps one or more of the models could be shown to be internally inconsistent, or problematic in some other way. I cannot rule this out. But the anti-Cartesian considerations described above suggest that we should consider another possibility: that the issue over which the cinematic theorists, retentional theorists and extensional theorists are disagreeing is entirely 
chimerical. If we relax the assumption that there is a definite moment at which information "enters" or "leaves" consciousness, or that a conscious experience starts and ends at determinate moments in time, then we can describe temporal experience in a different way. Perhaps we might think of the question of when information enters consciousness as comparable to the question of when exactly some information has been received by a large organization such as the BBC. The information might reach different parts of the organization at different times, with no principled reason to pick one of them as more important than the others. Following this line of thought, we might eschew the notion of an experience and say instead, for example, that there are long periods during which a subject is experiencing the world, and that during such a period - a period that does not start at a precise, determinate instant - the subject becomes aware that $\mathrm{A}$ is followed by $\mathrm{B}$, which is followed, by $\mathrm{C}$, and so on. There is indeed something that it is like for the subject during that episode of conscious experience, but what it is like is perhaps exhausted by what the subject experiences (the various events, and the sequence in which they occur). There need be nothing more to say than that.

\section{References}

Anstis, S., Verstraten, A. J., and Mather, G. (1998). "The Motion Aftereffect," Trends in Cognitive Sciences 2: 111-117.

Arstila, V. (2016) "The Time of Experience and the Experience of Time", in Bruno Mölder, Valtteri Arstila and Peter Øhrstrøm (eds.) Philosophy and Psychology of Time, vol.9 of the series Studies in Brain and Mind. Dordrecht: Springer.

Broad, C. D. (1923) Scientific Thought, London: Kegan Paul.

Broad, C. D. (1938) An Examination of McTaggart's Philosophy (Volume II, Part 1), Cambridge: Cambridge University Press.

Casati, R., and Dokic, J. (1994) La philosophie du son, Nîmes: Chambon.

Chuard, P. (2011) “Temporal Experiences and Their Parts," The Philosophers' Imprint 11: $1-28$.

Dainton, B. (2000) Stream of Consciousness: Unity and Continuity in Conscious Experience, London: Routledge.

Dainton, B. (2001) Time and Space, Durham: Acumen. Second edition 2010.

Dainton, B. (2008) "Sensing Change," Philosophical Issues 18: 362-384.

Dainton, B. (2010) “Temporal Consciousness," in E. N. Zalta (ed.) The Stanford Encyclopedia of Philosophy (Fall 2010 Edition). Online at http://plato.stanford.edu/archives/fall2010/entries/consciousness-temporal/. 
Dennett, D. C. (1988) "Quining Qualia," in A. Marcel and E. Bisiach (eds.) Consciousness in Contemporary Science. Oxford: Oxford University Press.

Dennett, D. C. (1991) Consciousness Explained, London: Penguin.

Foster, J. (1979). "In Self-Defence," n G. F. Macdonald (ed.) The Problem of Pure Consciousness, London: Macmillan.

Foster, J. (1982) The Case for Idealism, London: Routledge and Kegan Paul.

Foster, J. (1991) The Immaterial Self, London: Routledge.

Grush, R. (2007) "Time and Experience," in T. Müller (ed.) The Philosophy of Time, Frankfurt: Klosterman.

Hirsh, I. J. and Sherrick, C. E. (1961) "Perceived Order in Different Sense Modalities," Journal of Experimental Psychology 62: 423-432.

Hoerl, C. (2009) “Time and Tense in Perceptual Experience," Philosophers' Imprint 9: $1-18$.

Hoerl, C. (2013) "A Succession of Feelings, in and of itself, is Not a Feeling of Succession," Mind 122: 373-417.

James, W. (1890) The Principles of Psychology, New York: Henry Holt. Reprinted Cambridge, MA: Harvard University Press, 1983.

Le Poidevin, R. (2007) The Images of Time, Oxford: Oxford University Press.

Nagel, T. (1974) "What is it Like to Be a Bat?," The Philosophical Review 83: 435-450.

O'Callaghan, C. (2007) Sounds, Oxford: Oxford University Press.

Pasnau, R. (2007) “The Event of Color", Philosophical Studies 142: 353-369.

Phillips, I. (2010) "Perceiving Temporal Properties," European Journal of Philosophy 18: 176-202.

Phillips, I. (2011) "Indiscriminability and Experience of Change," The Philosophical Quarterly 61: 808-827.

Phillips, I. (2014) "The Temporal Structure of Experience," in D. Lloyd and V. Arstila (eds.), Subjective Time: The Philosophy, Psychology, and Neuroscience of Temporality, Cambridge, MA: MIT Press 139-158.

Pockett, S. (2003) "How Long is 'Now'? Phenomenology and the Specious Present," Phenomenology and the Cognitive Sciences 2: 55-68.

Pöppel, E. (1997) "A Hierarchical Model of Temporal Perception," Trends in Cognitive Sciences 1: 56-61.

Prosser, S. (2016) Experiencing Time, Oxford: Oxford University Press.

Rashbrook, O. (2013) “An Appearance of Succession Requires a Succession of Appearances," Philosophy and Phenomenological Research 87: 584-610.

Rensink, R. A. (2002) "Change Detection," Annual Review of Psychology 53: 245-77.

Rensink, R. A., O’Regan, J. K., Clark, J. J. (1997) "To See or Not to See: The Need for Attention to Perceive Changes in Scenes," Psychological Science 8: 368-73.

Ruhnau, E. (1995) “Time-Gestalt and the Observer”, in T. Metzinger (ed.) Conscious Experience, Paderborn: Ferdinand Schöningh/Imprint Academic: 165-184.

Simons, D. J., and Levin, D. T. (1997) “Change Blindness," Trends in Cognitive Science 1: 261-67.

Turatto, M., Bridgeman, B. (2005) "Change Perception Using Visual Transients: Object Substitution and Deletion,” Experimental Brain Research 167: 595-608.

Wohlgemuth, A. (1911) On the After-Effect of Seen Movement: Volume 1, Cambridge: Cambridge University Press. 